\section{Fatores ergonômicos de risco e de proteção contra acidentes de trabalho: um estudo caso-controle}

\section{Ergonomic risk and protection factors from health accidents: a case-control study}

\author{
Raphael Mendonça Guimarães ${ }^{1 *}$ \\ Maria Yvone Chaves Mauro² \\ René Mendes ${ }^{3}$ \\ André Oliveira de Melo ${ }^{4}$ \\ Tatiana Fernandes da Costa \\ 'Enfermeiro residente em Saúde Pública da Secretaria de Estado da Saúde \\ ${ }^{2}$ Departamento de Enfermagem em Saúde Pública, Universidade do Estado \\ do Rio de Janeiro \\ ${ }^{3}$ Departamento de Saúde Pública, Faculdade de Medicina da Universidade \\ Federal de Minas Gerais \\ ${ }^{4}$ Programa de Saúde da Família de Belo Horizonte \\ ${ }^{5}$ Instituto Estadual de Hemoterapia do Estado do Rio de Janeiro (HEMORIO)
}

\section{Resumo}

O presente trabalho tem como objetivo estudar a relação entre os acidentes ocupacionais e os riscos ergonômicos no âmbito da organização do processo de trabalho de Enfermagem. Foi utilizado o método epidemiológico e um desenho de estudos casocontrole. O universo de estudo foram enfermarias do serviço de enfermagem clínica do Hospital Universitário Pedro Ernesto (HUPE), localizado no município do Rio de Janeiro. Os dados foram analisados com o programa Epi-Info $2004^{\circledR}$, onde foram comparados, através de uma análise de odds ratio, os fatores estudados em um grupo controle de não acidentados e um grupo de estudo de acidentados. Obtivemos, como resultado, variáveis que foram classificadas por grupos de risco e proteção, de acordo com os valores de medidas encontrados. Foram considerados fatores de risco: divisão de tarefas insatisfatórias, concentração de atividades excessiva, acúmulo de divisão de tarefas, atividades de crescimento profissional, ocupação total da carga horária durante a jornada de trabalho. Como fatores de proteção: pausas durante o trabalho, disponibilidade de EPI, utilização de EPI, compatibilidade entre o cargo e o maior nível de formação, retorno da chefia quanto ao desempenho exercido e realização profissional.

Palavras-Chave: Saúde ocupacional. Acidente de trabalho. Epidemiologia.

*Correspondência: Rafael Mendonça, Travessa Alfredo Botelho, 52, 20720-200Méier, Rio de Janeiro, 


\section{Abstract}

The research aimed to study the relationship between occupational accidents and ergonomic risks in the nursing work process. The epidemiological method was used with a case-control design. The research site was the clinical ward of Pedro Ernesto Academic Hospital in Rio de Janeiro. Data were analyzed with Epi-Info $2004^{\circledR}$. Factors in the case group of workers who had an accident and the control group of workers who did not have an accident were compared through odds ratio. As results, we attained variables classified into risk groups and protection groups, according to the measurements found.

Key Words: Occupational health. Work accidents. Epidemiology.

\section{Introdução}

A Saúde do Trabalhador é um campo específico da área da saúde pública que procura atuar através de procedimentos próprios com a finalidade de promover e proteger a saúde de pessoas envolvidas no exercício do trabalho ${ }^{1}$. Isto implica em uma atuação multidisciplinar e interdisciplinar em que a enfermagem está inserida, junto a outros profissionais especializados, buscando a preservação e a promoção da saúde através de medidas de alcance coletivo ${ }^{2}$.

Um ponto importante diz respeito ao processo gerador saúde-doença, ao qual os trabalhadores da saúde estão expostos. Estes contemplam variados riscos e fatores predisponentes ao desequilíbrio biopsicossocial. Tais riscos e fatores em grande parte não são encarados com a seriedade que deveriam e, com isso, geram muitas vezes, silenciosamente, os agravos à saúde ${ }^{1}$.

Considera-se, para este estudo, que a eficiência, a eficácia e o bem-estar do sujeito no contexto de trabalho dependem fundamentalmente da capacidade de regulação da atividade laboral com dupla finalidade: gerir as variações das condições externas e internas da atividade e controlar os seus efeitos. Neste sentido, este estudo está justificado. Sendo assim, tem-se como objeto de estudo os acidentes de trabalho ocorridos em um Hospital Universitário do Estado do Rio de Janeiro. Para tal investigação, traçou-se como objetivo realizar análise comparada entre o grupo caso de acidentados e um grupo controle de não acidentados emparelhados por sexo, ocupação, turno de trabalho, tempo na função e outros vínculos empregatícios.

\section{Metodologia}

Este é um estudo analítico e observacional, cujo desenho se enquadra no tipo caso-controle, segundo critérios de autoresreferência na Epidemiologia ${ }^{3-5}$.

Na medida do possível, a seleção de controles deve ser feita com o objetivo de obter uma amostra comparável dos casos, isto é, que pertençam à mesma população de base, 
para a qual posteriormente será feita a generalização das conclusões ${ }^{3}$.

Após a seleção dos participantes, pretende-se determinar, retrospectivamente, a freqüência de exposição aos fatores (variáveis independentes) que se pretende estudar, e se pensa estarem associados com o evento acidente de trabalho.

O estudo foi realizado no Hospital Universitário Pedro Ernesto (HUPE), localizado no Estado do Rio de Janeiro, em município do mesmo nome, no bairro de Vila Isabel, Área Programática 2.2 na IX Região Administrativa, no período de janeiro a novembro de 2004.

No presente estudo, a população utilizada foi a de trabalhadores de enfermagem do serviço de enfermagem clínica de um hospital universitário do Rio de Janeiro. Especificamente, lidamos com os trabalhadores das enfermarias de clínica médica e especialidades, totalizando 266 trabalhadores de ambos os sexos..

Foram avaliadas neste estudo variáveis direcionadas às condições de trabalho, norteadas pelo referencial teórico da ergonomia $^{6-8}$. O estudo de tais variáveis ocorreu pelo fato de se tratarem de características que se distinguem de uma visão puramente biologicista, e incorporarem uma visão de promoção da saúde ${ }^{9}$ no trabalho.

Em um primeiro momento, realizamos a coleta de dados através de formulários com questões fechadas, no campo escolhido, com toda a população de sujeitos, ou seja, com todos os trabalhadores das equipes de enfermagem lotadas nas enfermarias observadas. A seguir, fizemos um screening de indivíduos acidentados no período entre $1998 \mathrm{e}$ 2003, e de não acidentados, formando com isso dois grupos de sujeitos: os casos - aqueles que sofreram acidentes ocupacionais; e os controles - aqueles que não sofreram acidentes de trabalho.

Depois disso, quantificamos os dados referentes às características observadas e avaliadas pelos próprios trabalhadores, procedendo à análise a partir dos dados de freqüência comparados, utilizando a medida da razão de chances, ou odds ratio. Através desta me- dida de associação procuramos explorar as relações das características isoladas entre os grupos. A partir desta comparação, além de traçarmos uma análise pormenorizada das variáveis mais destacadas nas comparações, estimamos através da fórmula para cálculo da RAP (risco atribuível na população), segundo Levin ${ }^{3}$, qual seria o impacto sobre a freqüência dos acidentes, caso tal variável fosse trabalhada dentro de uma perspectiva de planejamento estratégico.

Em cumprimento à resolução de 196/96 ${ }^{10}$ do Conselho Nacional de Saúde, no mês de setembro o referido trabalho foi enviado ao Comitê de Ética em Pesquisa (CEP) do hospital em questão, para análise e autorização da coleta de dados nas unidades referidas e a utilização do nome da Instituição, autorização esta recebida no mês de outubro.

Os dados foram obtidos de trabalhadores do serviço de enfermagem clínica do Hospital Universitário Pedro Ernesto (HUPE), sendo selecionada aleatoriamente uma amostra composta das seguintes enfermarias/especialidades: Clínica Médica, DIP, Pneumologia, Cardiologia e Núcleo de Estudo da Saúde do Adolescente (NESA). O total de trabalhadores de enfermagem do serviço de clínica é 272. A totalidade das enfermarias adotadas como amostra contemplam 211 trabalhadores. A coleta teve um contingente diminuído devido a diversas situações, tais como: o período de greve, licença, férias e licença médica. Houve, dentro da amostra, uma perda de $33 \%$ dos formulários. Foram totalizados, então, 180 formulários devolvidos, com os quais processamos os números e obtivemos os dados.

Foram definidos como casos todos os que se acidentaram por razões de trabalho entre 1998 e 2003, dentro do Hospital Universitário, utilizando-se toda a amostra de acidentados para o estudo, o que totalizou 63 vítimas, perfazendo o total de $35 \%$ da amostra analisada.

Considerou-se, neste momento, as seguintes variáveis para comparação e montagem do grupo controle: sexo, ocupação, turno de trabalho, tempo de função exercida e se trabalha ou não em outra instituição 
Os grupos caso e controle constam, portanto, de 63 sujeitos cada, cujo perfil é de mulheres cujo vínculo de trabalho é o de auxiliar de enfermagem, em regime estatutário, trabalhando em serviço diurno, com tempo de função entre 10 e 15 anos, e que não trabalham em outra instituição. Foram excluídos os que trabalhavam em duas instituições.

Após a coleta, processou-se os dados através do programa Epi-Info® versão 3.3 de 2004, pelo qual se obteve a seqüência de variáveis que seriam estudadas.

Para a conclusão deste trabalho, propomos a obtenção de dados quantitativos acerca destes fatores. Nossa intenção foi determinar, a partir dos dados de freqüência da ocorrência do fator relacionado, e da sua respectiva $\mathrm{OR}$, qual seria a redução do risco de acidente. Para tal, utilizamos o que a Epidemiologia chama de Risco Atribuível nas Populações/RAP ${ }^{4}$. Segundo Medranho, a RAP é uma medida de impacto e uma estimativa da "quantidade de doença" que é atribuível, unicamente, à exposição. Representa, também, a proporção de doenças que poderiam ser eliminadas se fosse removida a exposição. Não há necessidade de se inserir duas páginas de texto sobre este método de análise.

Esta medida é obtida em percentuais, $\mathrm{e}$ pode ser calculada através de uma fórmula conhecida como Fórmula de Levin. Segundo esta fórmula, obtém-se o valor de RAP (\%) através da expressão:

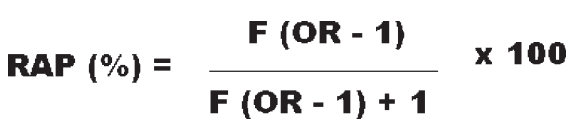

Fórmula de Levin (Medronho, 2003)

em que F é a proporção de ocorrência de um fator na população total, ou seja, a proporção da população exposta ao fator, e OR é a razão de chances/odds ratio.

A RAP é eficiente para o cálculo dos fatores de risco, mas não para os fatores de proteção, na medida em que, sendo a OR um valor abaixo de 1 para os fatores de proteção, o cálculo seria negativo. Sendo assim, utilizamos, para os fatores de proteção, a Fórmula de Levin adaptada por Guimarães, que segue a seguinte Lei:

\section{$\operatorname{RAP}(\%)=\frac{F(1-\text { OR })}{F(1-\text { OR })+1} \times 100$}

Fórmula de Levin adaptada por Guimarães

Calculou-se, ainda, o RAP segundo Levin para fatores de risco e de proteção, o que trouxe em números a relação entre os fatores e o acidente de trabalho. Matematicamente, a RAP determina que, se retirado o fator de risco $\mathrm{X}$, ou incluído o fator de proteção $\mathrm{Y}$, o número de acidentes de trabalho será reduzido em cada porcentagem.

\section{Resultados}

Seguem-se os gráficos referentes aos dados demográficos dos grupos caso e controle analisados.

\section{Dados dos Acidentes de Trabalho}

Constatou-se que a maior parcela de acidentados sofreu apenas um acidente (68\%), que em sua maioria ocorreu durante ou no momento de um procedimento (84\%). $\mathrm{Na}$ Tabela 1 observamos as características dos acidentes de trabalho (AT).

Pela própria característica da rotina de trabalho da enfermagem, as mãos são as partes do corpo mais utilizadas, e portanto mais expostas a eventuais acidentes, sejam eles com materiais perfurocortantes ou luxações, quebra, tendinites ou doenças ocupacionais. Os números encontrados ratificam essa circunstância (65\%).

Observa-se ainda que o grupo caso apresenta seu calendário vacinal mais atualizado do que o grupo controle, no que diz respeito às vacinas contra hepatite B e Tétano. Embora estatisticamente não haja uma diferença significativa, cabe ressaltar este fato, tendo em vista que ter um grupo acidentado mais coberto vacinalmente corrobora a hipótese de que os mesmos só tenham se vacinado após o acidente, devido à rotina de 


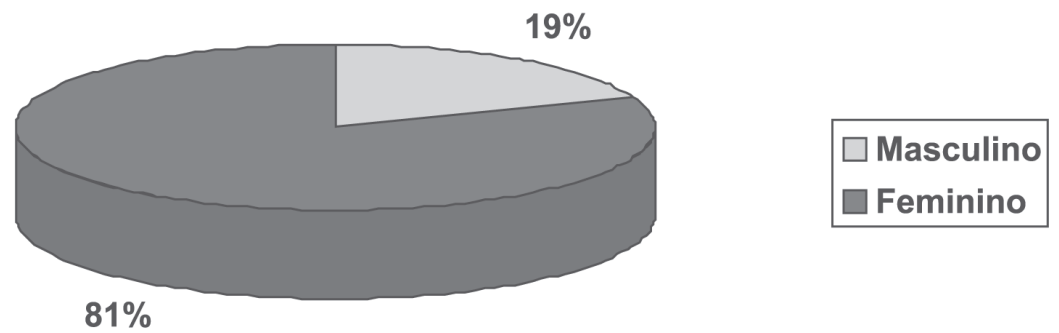

Gráfico 1- Distribuição de freqüência do grupo caso segundo sexo. HUPE/UERJ - Rio de Janeiro, 2004.

Chart 1 - Frequency distribution of the case group according to sex. HUPE/UERJ - Rio de Janeiro, 2004.

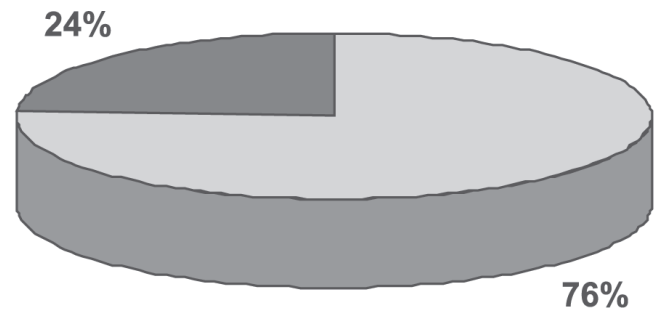

\section{Auxiliar de Enfermagem \\ $\square$ Enfermeiro}

Gráfico 2 - Distribuição de freqüência do grupo caso segundo ocupação. HUPE/UERJ - Rio de Janeiro, 2004.

Chart 2 - Frequency distribution of the case group according to occupation. HUPE/UERJ - Rio de Janeiro, 2004.

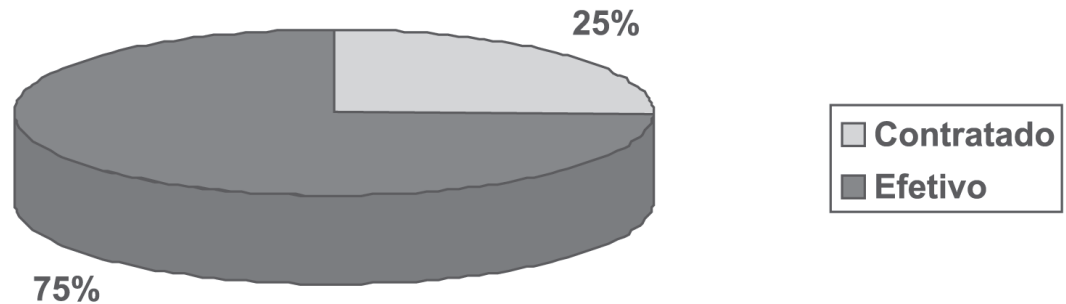

Gráfico 3 - Distribuição de freqüência do grupo caso segundo situação ocupacional. HUPE/ UERJ - Rio de Janeiro, 2004.

Chart 3 - Frequency distribution of the case group according to occupational status. HUPE/UERJ - Rio de Janeiro, 2004.

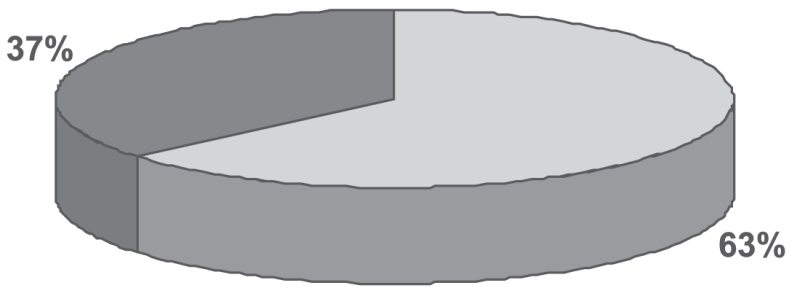

$\square$ Diurno

Noturno

Gráfico 4 - Distribuição de freqüência do grupo caso segundo turno de trabalho. HUPE/UERJ - Rio de Janeiro, 2004.

Chart 4 - Frequency distribution of the case group according to work shift. HUPE/UERJ - Rio de Janeiro, 2004. 


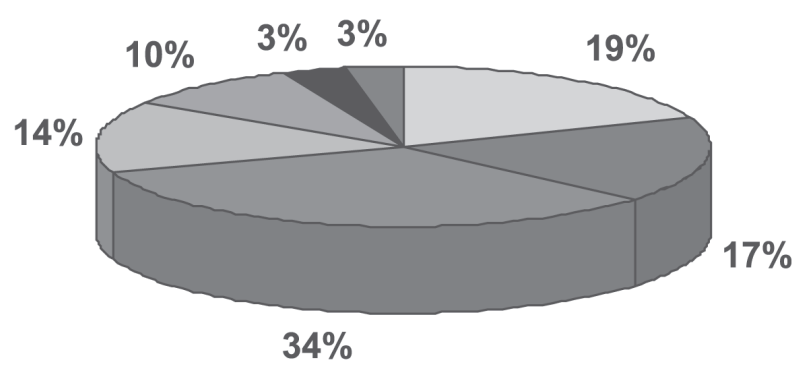

\begin{tabular}{|l|}
\hline 0-5 anos \\
$\square$ 5-10 anos \\
$\square 10-15$ anos \\
$\square 15-20$ anos \\
$\square 20-25$ anos \\
$\square 25-30$ anos \\
$\square+$ de 30 anos
\end{tabular}

Gráfico 5 - Distribuição de freqüência do grupo caso segundo tempo de função exercida. HUPE/UERJ - Rio de Janeiro, 2004.

Chart 5 - Frequency distribution of the case group according to time in the job. HUPE/UERJ - Rio de Janeiro, 2004.
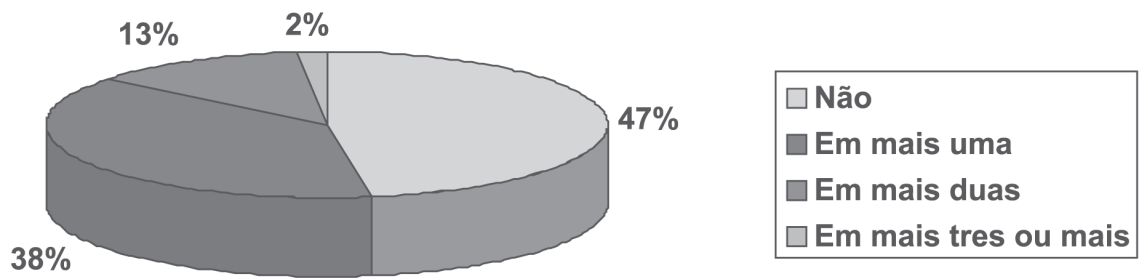

Gráfico 6 - Distribuição de freqüência do grupo caso segundo trabalho ou não em outra instituição de saúde. HUPE/UERJ - Rio de Janeiro, 2004.

Chart 6 - Frequency distribution of the case group according to work in other health institution. HUPE/ UERJ - Rio de Janeiro, 2004.

Tabela 1 - Variáveis e Suas Medidas de Associação. HUPE/UERJ - Rio de Janeiro, 2004

Table 1 - Variables and Association Measurements. HUPE/UERJ - Rio de Janeiro, 2004.

\begin{tabular}{lcc}
\hline Variável & Odds Ratio & IC (95\%) \\
\hline Espaço físico de trabalho inadequado & 0,72 & $0,36-1,47$ \\
Disposição de mobiliário inadequada & 0,82 & $0,40-1,68$ \\
Divisão de tarefas insatisfatória & 3,18 & $1,47-6,89$ \\
Realização de pausas durante o trabalho & 0,31 & $0,13-0,78$ \\
Concentração de atividadesexcessiva & 3,02 & $1,36-6,72$ \\
Disponibilidade de recursos materiais & 0,68 & $0,28-1,62$ \\
Atividades designadas excessivas & 1,67 & $0,82-3,37$ \\
Disponibilidade de EPI & 0,46 & $0,23-0,94$ \\
Utilização de EPI & 0,41 & $0,19-0,88$ \\
Satisfação com remuneração/ salário & 1,44 & $0,68-3,04$ \\
Equilíbrio na remuneração entre funcionários da mesma categoria & 0,48 & $0,11-1,99$ \\
Atividade exercida compatível com maior nível de formação & 0,38 & $0,17-0,86$ \\
Autonomia no trabalho & 1,21 & $0,51-2,88$ \\
Participação nas decisões de trabalho & 0,93 & $0,40-1,98$ \\
Acúmulo concomitante de tarefas & 2,24 & $1,08-4,66$ \\
Retorno da chefia quanto ao desempenho exercido & 0,40 & $0,19-0,85$ \\
Realização de atividades de crescimento profissional & 2,20 & $1,07-4,52$ \\
Satisfação na vida familiar & 0,63 & $0,21-1,89$ \\
Relacionamento interpessoal favorável & 0,87 & $0,31-2,43$ \\
Realização de atividades de lazer & 0,68 & $0,31-1,47$ \\
Existência de normas e regulamentos acessíveis & 0,83 & $0,41-1,66$ \\
Apoio dos colegas na realização de tarefas & 3,10 & $0,31-30,64$ \\
Ocupação total da carga horária durante a jornada de trabalho & 2,55 & $1,23-5,29$ \\
Realização Profissional & 0,31 & $0,13-0,78$ \\
\hline
\end{tabular}


vacinação que é feita em caso de acidentes. Podemos trazer também como hipótese que os mesmos não são tão assíduos às campanhas de vacinação, estando com isso mais suscetíveis a contrair estas doenças.

Os dados referentes às variáveis estudadas comparando-se o grupo caso e o grupo controle encontram-se na Tabela 1:

Visto isso, para efeitos de discussão, realizamos um recorte que definiu, com os critérios empregados, 11 variáveis sendo 5 agressoras e 6 protetoras. Vale lembrar que as demais variáveis são importantes no sentido de sinalizar deficiências, algumas delas graves, do próprio sistema administrativo e da condução de gerência da enfermaria e do hospital como um todo, porém não abarcam a temática do estudo proposto.

A seguir observamos, já com os números apurados e submetidos a um processamento estatístico, qual o impacto de tais fatores na ocorrência de acidentes de trabalho. Tal cálculo foi realizado através da RAP citada anteriormente. Nas Tabelas 2 e 3 podemos observar os respectivos valores de OR e RAP.

\section{Discussão}

A maneira verdadeiramente eficaz de impedir o acidente é conhecer e controlar os riscos. Consideramos fundamental para a avaliação dos fatores preditivos de acidentes de trabalho, seja de risco ou de proteção, a contribuição da Ergonomia Francesa ou Contemporânea como instrumento teóricometodológico voltado para o estudo da relação trabalho e saúde ${ }^{7}$. Por ser uma área interdisciplinar, onde se articulam os conhecimentos da fisiologia, da psicologia, da sociologia e antropologia, a Ergonomia Francesa tem sido utilizada com freqüência nas análises dos acidentes. A Ergonomia é um instrumento que busca conhecer o trabalho humano, preconizando para isso a observação dos trabalhadores em situação de trabalho. A fala no ambiente de trabalho tem um extremo valor para a análise da atividade.

A partir da análise realizada, com a obtenção dos dados de odds ratio e o intervalo de confiança, pudemos estabelecer que variáveis seriam interessantes para uma análi-

Tabela 2 - Fatores de Risco e suas Medidas de Associação/ Impacto. HUPE/UERJ - Rio de Janeiro, 2004.

Table 2 - Risk Factors and Association/Impact Measurements. HUPE/UERJ - Rio de Janeiro, 2004.

\begin{tabular}{lccc}
\hline Fator de Risco & $\mathrm{F}$ & $\mathrm{OR}$ & $\mathrm{RAP}$ \\
\hline Divisão de tarefas insatisfatórias & 0,65 & 3,18 & 0,59 \\
Concentração de atividades excessiva & 0,71 & 3,02 & 0,59 \\
Acúmulo de divisão de taferas & 0,60 & 2,24 & 0,43 \\
Atividades de crescimento profissional & 0,57 & 2,20 & 0,41 \\
Ocupação total da carga horária durante a jornada de trabalho & 0,59 & 2,55 & 0,48 \\
\hline
\end{tabular}

Tabela 3 - Fatores de Proteção e suas Medidas de Associação/ Impacto. HUPE/UERJ - Rio de Janeiro, 2004.

Table 3 - Protection Factors and Association/Impact Measurements. HUPE/UERJ - Rio de Janeiro, 2004.

\begin{tabular}{lccc}
\hline Fator de Proteção & $F$ & OR & RAP \\
\hline Pausas durante o trabalho & 0,78 & 0,31 & 0,35 \\
Disponibilidade de EPI & 0,52 & 0,46 & 0,22 \\
Utilização de EPI & 0,68 & 0,41 & 0,29 \\
Compatibilidade entre o cargo e o maior nível de formação & 0,71 & 0,38 & 0,30 \\
Retorno da chefia quanto ao desempenho exercido & 0,64 & 0,40 & 0,28 \\
Realização profissional & 0,78 & 0,31 & 0,35 \\
\hline
\end{tabular}


se do risco e da proteção de acidentes de trabalho.

\section{Fatores de risco}

Dados os resultados, obtivemos os seguintes fatores de risco para discussão:

\section{Divisão de tarefas insatisfatórias}

A divisão de tarefas foi tida como fator de risco potencial, tendo o profissional um risco 3,18 vezes maior de sofrer acidentes quando realiza divisão de tarefas. Esta divisão é quase rotineira em serviços de enfermagem, especialmente entre a equipe de auxiliares, que são divididos em atividades de higiene, medicação, controle de diurese e sinais vitais. Atribui-se como hipótese para este risco o fato de que, com esta divisão, e sem um rodízio desta divisão, há a mecanização do trabalho, o que, além de atribuir ao processo de trabalho um caráter alienador, contribui para que os profissionais, julgando-se aptos para aquele serviço, executem-no sem tomar medidas adequadas de prevenção de acidentes, como o uso de luvas para coleta de sangue ou a solicitação de auxílio para mobilização do paciente no leito.

Cabe destacar que a divisão de tarefas se faz necessária principalmente por haver um contingente insuficiente de trabalhadores nas equipes, obrigando o grupo a otimizar o tempo para poder cumprir as atividades - como uma "automação do cuidado" -, haja vista a forma desumanizada e fragmentada que este cuidado transmite ${ }^{6,11}$. Este déficit de pessoal, como veremos a seguir, interfere ainda em outros fatores de risco.

\section{Excessiva concentração das atividades}

A concentração das atividades em determinado horário mostrou ser capaz de aumentar em 3,02 vezes a probabilidade de ocorrência de acidentes. Considera-se, para efeito de análise, que esta concentração se dá em horários próprios de picos de sobrecarga da equipe, que muitas vezes existe em função da rigidez da rotina do serviço. Um exemplo claro desta situação é, por exemplo, o horário das 10 horas da manhã em um setor de enfermagem. Nesse momento, há a situação da realização de todos os banhos de leito, administração de etapas de antibioticoterapia, hidratação venosa, aferição de sinais vitais, rotinas administrativas como requisição ou recepção de material, e evolução dos pacientes.

Na verdade, este padrão estabelecido de horários para as tarefas não é, nem nunca foi, uma regra ou lei sujeita a penalidades, mas é cristalizado no processo de trabalho e há muito tempo se percebe um desgaste do profissional nesse horário de pico, além de criar um ambiente de trabalho inóspito, com concentração de pessoas circulantes na enfermaria, ruídos excessivos e estresse por tentar cumprir as metas. Portanto, a flexibilidade neste padrão é uma forma bastante apropriada de diminuir este risco de acidente ${ }^{7,12,14}$.

\section{Acúmulo concomitante de tarefas}

O profissional que realiza mais de uma tarefa ao mesmo tempo estará sujeito 2,24 vezes mais a se expor a um acidente. Isto está ligado ao fato de que, ao realizar o trabalho desta forma, o profissional não está atento aos próprios limites humanos quando se executa qualquer tarefa, já que este acúmulo de tarefas exige mais esforço do que o normal. Falamos aqui de limites tanto físicos quanto fisiológicos, ou mentais e emocionais $^{6,11}$.

Não é cabível, por exemplo, exigir que uma pessoa execute duas tarefas em que seja exigido um máximo de concentração para cada uma delas, como verificar a evolução de um paciente e fazer pedidos ao mesmo tempo. Vale a pena destacar, neste momento, que mais uma vez a carência de número satisfatório de trabalhadores repercute negativamente para esta situação, na medida em que não seria necessário este acúmulo caso houvesse pessoal suficiente.

\section{Atividades de crescimento profissional}

Curiosamente, esta variável entrou para uma classificação de risco no local do nosso estudo. Como é costume perceber, atividades de educação continuada ou permanente nos hospitais são apontadas como incen- 
tivo à qualificação dos trabalhadores, maior rendimento profissional e garantia de qualidade da assistência. Entretanto, aqui, mostra-se que o grupo que sofreu acidente é exatamente o que passou por mais processos de atualização e/ou aperfeiçoamento. Tentamos simular uma hipótese para este quadro: a educação continuada que vem sido ofertada a este grupo estará equivocada? Será que de fato ela atenta para as necessidades reais das equipes? Tem sido necessário a este grupo procurar este serviço fora do próprio hospital?

Estas hipóteses, impossíveis de serem julgadas ou testadas neste momento, só vêm reforçar a necessidade de se superar um paradigma de educação continuada nos serviços, que se vale tão somente das oportunidades que surgem para a realização de atividades, e de se realizar uma educação permanente, que se distância não só no nome, mas no propósito. Isto significa um processo contínuo de aperfeiçoamento, mas com uma forma de ligação com o contexto do trabalho e com as necessidades locais, sem fórmulas prontas.

\section{Ocupação total da carga horária durante a jornada de trabalho}

Finalmente, temos um último fator de risco sinalizado, que seria a ocupação total da carga horária durante a jornada, ou seja, estar em atividade ao longo de todo o plantão. Este fator de risco, quando presente, mostra uma associação negativa de 2,55 vezes maior à probabilidade de acidentes. Assim como outros fatores já citados, este também está relacionado à escassez de pessoal, que impede que este trabalhador possa realizar pausas durante a jornada, uma condição de proteção que será discutida posteriormente ${ }^{7,13}$.

\section{Fatores de Proteção}

De acordo com a análise estatística empregada, obtivemos os seguintes fatores de proteção:

\section{Realização de pausas durante o serviço Um dos eixos em que a ergonomia tor-}

na-se aliada da saúde do trabalhador está no fato de considerar que o trabalho deve se ajustar ao trabalhador, não o contrário ${ }^{15,16}$.

Isto é possível mediante modificações no sentido de eliminar esforço excessivo e posturas incômodas, e reduzir movimentos repetitivos. Um excelente exemplo disto, e ratificado neste estudo, é proporcionar pausas de descanso para aliviar grupos musculotendinosos fatigados. O tempo necessário também não deverá ser predeterminado, dependendo exclusivamente do esforço global de trabalho e da duração do ciclo/jornada de trabalho.

\section{Disponibilidade de EPI}

A disponibilidade de EPI é um dado importante no que se refere à prevenção de acidentes. Os EPIs devem estar disponíveis no local onde são necessários, e o treinamento especial para seu uso deve ser ministrado principalmente para os profissionais de apoio.

Além disso, ao selecionar o equipamento de proteção individual (EPI) deve-se prestar atenção aos agentes de estresse. O EPI deve existir em diversos tamanhos, deve satisfazer as características físicas dos trabalhadores e do trabalho e, mais importante, não deve contribuir para posturas extremas e esforço excessivo ${ }^{15}$.

\section{Utilização de EPI}

O clima institucional e o apoio gerencial têm um papel importante na adequação entre o treinamento e a aderência às recomendações, destacando-se a importância dos supervisores na orientação e reforço das práticas adequadas. Além disso, é válido ressaltar o que foi dito anteriormente, sobre o fato de o EPI não dever contribuir para posturas extremas e esforço excessivo.

Na verdade, o EPI é direito de qualquer trabalhador, mas não deve servir de medida paliativa para mascarar uma condição inapropriada de trabalho, como, por exemplo, o uso de máscaras tipo N95, conhecida pela proteção que confere contra bacilos de Kock (que causam tuberculose) em enfermarias de Doenças Infectoparasitárias, por- 
que a unidade não dispõe de leitos de precaução respiratória. Enfim, uma medida não deve ser substituta à outra, mas complemen$\operatorname{tar}^{16}$.

Os próximos fatores de proteção, embora destacados, relacionam-se à satisfação que se obtém através do trabalho. A satisfação no trabalho é um estado de prazer emocional que ocorre devido a duas variáveis: valores e interesses que são da própria personalidade do indivíduo e refletem a sua vocação, o que ele gosta de fazer e a avaliação que faz da própria natureza do cargo; benefícios tangíveis que a empresa oferece e fazem o indivíduo trabalhar melhor, como um bom salário, qualidade da chefia, equipamentos e infra-estrutura adequada. As empresas sempre encontrarão dificuldades para motivar todos os empregados. Cada ser humano tem sua escala de valores, suas expectativas, seus sonhos e necessidades diferentes. Além disso, a natureza humana é marcada pela insatisfação, e esta mobiliza posturas e estimula a ação e a transformação. Pode-se ampliar os níveis de satisfação, mas eliminar por completo a insatisfação não parece uma medida possível ${ }^{8,11}$.

\section{Compatibilidade de atividade exercida com maior nível de formação}

A possibilidade de mobilidade horizontal (quadro de carreira), com o respectivo aumento salarial, além da possibilidade de treinamento e reciclagem, trazem satisfação no trabalho. Não foi incomum, no momento da coleta de dados, encontrarmos profissionais com cargo inferior ao seu maior nível de formação (por exemplo, auxiliares de enfermagem que já possuem nível superior completo, mas permanecem exercendo atividades de auxiliar de enfermagem). Este era um fator ansiogênico, já que, como auxiliar, o profissional não pode interferir em certas condutas ou realizar certas atividades por impedimento da própria chefia, que pode ser inflexível, ou por aspectos legais, pois isto configuraria um deslocamento de função sem adicional salarial para isto.

Tal quadro nos traz a comprovação de que, quando o profissional realiza suas tare- fas com plena propriedade para a qual teve formação/capacitação, estas atividades são mais bem recebidas pela pessoa, que se sente valorizada por sua qualificação profissional. Este fator elimina boa parte do estresse e da insatisfação no trabalho, que eventualmente poderiam propiciar um acidente ocupacional $^{11,15,17}$.

\section{Retorno da chefia quanto ao desempenho exercido}

Para ter satisfação, o funcionário também precisa entender quais os resultados da sua tarefa para a empresa. É muito fácil estabelecer metas e objetivos quase inatingíveis, mas ninguém se lembra de dar o feedback, etapa muito importante para o colaborador sentir-se reconhecido, valorizado e, portanto, satisfeito. ${ }^{12,14}$

Sentindo-se satisfeito, o trabalhador irá se empenhar cada vez mais para realizar as tarefas da maneira mais correta possível, tendo em vista que é valorizado por isso. Tratase de uma retroalimentação, que só contribui para que as atividades sejam realizadas com empenho e qualidade, o que dificulta a ocorrência de acidentes.

\section{Realização profissional}

Para que haja plena realização profissional, ou seja, para que o profissional esteja bem resolvido com sua tarefa e a desenvolva de forma prazerosa, é necessário um conjunto de condições favoráveis a isso: um grupo que esteja trabalhando na solução de um problema ou a formação de uma própria equipe; os meios de funcionamento como o elo entre os clientes; ter colegas, clientes ou um chefe dispostos a oferecer uma opinião objetiva sobre algo específico; uma oportunidade de fazer algo que exija muito do indivíduo (por exemplo, falar com os diretores, comandar uma força-tarefa, fazer um trabalho para uma nova área); um modo de variar a programação, o local ou as tarefas do trabalho diário; um modo de obter informações sobre o processo de trabalho antes das resoluções finais.

Estas condições determinarão o nível de adesão do trabalhador ao seu ofício, e esta re- 
alização é indispensável para que o trabalho seja realizado da forma correta, afastando com isso riscos de acidentes de trabalho ${ }^{12,17}$.

\section{Conclusão}

Percebeu-se, pela discussão, que há coerência entre os fatores estudados, na medida em que, internamente aos grupos de risco e proteção, os fatores se inter-relacionam. Além disso, alguns fatores de risco se contrapõem diretamente aos outros fatores de proteção, o que só ratifica sua classificação desta forma.

Neste sentido, fica como colaboração deste estudo os indicativos que podem alterar numericamente o número de acidentes de trabalho que, no local de estudo, mais uma vez ratificamos que se encontra em quantidade demasiadamente elevada ${ }^{17}$.

Lembramos, ainda, que tais fatores, na medida em que se relacionam com condições de trabalho, requerem uma mudança cultural da instituição, no sentido de valorizar mais os trabalhadores que a compõem, dando-lhes subsídios para um trabalho digno, decente, que satisfaça a necessidade de sua clientela e que, ao mesmo tempo, não seja danoso para quem executa as tarefas.

Cabe ressaltar a importância da satisfação no trabalho, ponto tão discutido pela ergonomia, que se aplica intimamente à área da saúde, fato bem marcado pelas conclusões deste estudo ${ }^{15}$.

\section{Referências}

1. Bulhões I. Enfermagem do trabalho. Volume 2. Rio de Janeiro: Idéias; 1986.

2. Minayo-Gómez C.; Thedim-Costa SM. A construção do campo de saúde do trabalhador: percurso e dilemas. Cad Saúde Pública 1997; 13(2): 21-32. .

3. Rouquayrol MZ, Almeida Filho N. 1999. Desenhos de Pesquisa em Epidemiologia. In: Rouquayrol MZ, Almeida Filho N. Epidemiologia e Saúde. (5a ed.) Rio de Janeiro: Medsi; 1999. p. 141-8.

4. Medronho R. Epidemiologia.. Rio de Janeiro: Atheneu; 2003.

5. Pereira MG. Epidemiologia: teoria e prática. (2 ${ }^{\mathrm{a}}$ ed) Rio de Janeiro: Guanabara Koogan; 2000.

6. Mauro MYC. Riscos Ocupacionais em Saúde. Rev Enfermagem Científica 1991; 3(I):11- 15.

7. Estryn-Behar M, Poinsignon H. Travailler à l'hospital. Paris: Berger Levrault; 1996.

8. Vidal MCR. Ergonomia na Empresa: Útil, Prática e Aplicada. Coleção Trabalho. Rio de Janeiro: Virtual Científica; 2001.

9. Buss PM. Promoção da saúde e qualidade de vida. Ciência \& Saúde Coletiva 2000; 5: 163-77.

10. Brasil. Ministério da Saúde. Conselho Nacional de Saúde. Resolução 196/96: Diretrizes e Normas Regulamentadoras de Pesquisas Envolvendo Seres Humanos. Brasília, DF. Disponível em < http:// www.bioetica.ufrgs.br/res19696.htm>. Acesso em 18 de novembro de 2004.
11. Mauro MYC, Guimarães RM, Muzi CD, Mauro CCC. Riscos Ocupacionais em Saúde. Rev Enfermagem UERJ 2004; 12(3): 316-22.

12. Almeida MCP, Rocha SMM. O trabalho de enfermagem. São Paulo: Cortez; 1997.

13. Braga D. Acidente de trabalho com material biológico em trabalhadores da equipe de enfermagem do Centro de Pesquisas Hospital Evandro Chagas. [dissertação de mestrado] Rio de Janeiro: Fundação Oswaldo Cruz, Escola Nacional de Saúde Pública; 2000.

14. Mendes R, Dias EC. Da medicina do trabalho à saúde do trabalhador. Revista de Saúde Pública 1991; 25(5): 341-49.

15. Fialho F, Santos N. Manual de Análise Ergonômica no Trabalho. Curitiba: Gênesis;1995.

16. Guimarães RM, Mauro MYC, Mauro CCC. Gestión del trabajo en perspectiva de ergonomia. In: Proceedings of the 3rd. Conference on occupational risk prevention. Santiago de Compostella; 2004.

17. Facchini LA. Uma contribuição da epidemiologia: o modelo da determinação social aplicado à saúde do trabalhador. In: Rocha LE, Rigotto RM, Buschimelli JTP (Org.). Isto é trabalho de gente? Vida, doença e trabalho no Brasil. Petrópolis: Vozes; 1994. p. 178-86.

recebido em: 29/03/05 versão final reapresentada em: 09/08/05 aprovado em: 11/08/05 


\section{INSTRUMENTO DE COLETA DE DADOS}

1. Cargo: ( ) Auxiliar de enfermagem ( ) Técnico de enfermagem ( ) Enfermeiro

2. Situação Ocupacional:

( ) Efetivo ( ) Cooperativado/Contrato ( ) Estagiário

3. Tempo de função:

( ) 0-5 anos ( ) 5-10 anos ( ) 10-15 anos ( ) 15-20 anos ( ) 20-25 anos

( ) 25-30 anos ( ) + de 30 anos

4. Sexo: ( ) masculino ( ) feminino

5. Turno de trabalho: ( ) diurno ( ) noturno

6. Trabalha em outra instituição de saúde? ( ) não ( ) sim. Quantas?

( ) em mais uma ( ) em mais duas ( ) em mais três ou mais

7. Já sofreu algum acidente de trabalho?

( ) não

( ) sim Quantas vezes? ( ) uma ( ) duas ( ) três ou mais

Horário do (s) acidente (s)?

Local(is) do corpo afetado(s) em cada acidente?

7.1 Foi no momento de algum procedimento: ( ) sim ( ) não

7.2 Você consegue relacionar o(s) acidente(s) a algum acontecimento na sua vida:

( ) $\operatorname{sim}(\quad)$ não

8. Caso tenha sofrido algum acidente, você realizou a notificação? ( ) sim ( ) não

9. Dentre os itens abaixo, assinale aqueles que você considera que trazem dificuldades para a realização do seu trabalho.

9.1 Organização do trabalho

- Espaço físico do trabalho: ( ) adequado ( ) não adequado

- Disposição do mobiliário: ( ) adequado ( ) não adequado

- Divisão de tarefas: ( ) satisfatória ( ) não satisfatória

- Realiza pausas durante o trabalho? ( ) sim ( ) não

- Existe concentração de atividades em determinado horário? ( ) não ( ) sim. Qual a hora?

- Disponibilidade de materiais permanentes e de consumo:

( ) suficiente ( ) insuficiente

- Número de atividades designadas é excessiva? ( ) sim ( ) não

9.2 Segurança do Trabalho

- Existe a disponibilidade de Equipamentos de Proteção Individual (EPI):

( ) $\operatorname{sim}(\quad)$ não

- Você utiliza o EPIs: ( ) sim ( ) não

- Calendário Vacinal (Hepatite B, DT, Hib), apresenta-se em dia?

( ) $\operatorname{sim}($ ) não

\subsection{Remuneração}

- Remuneração/Salário: ( ) adequado ( ) inadequado

- Há equilíbrio das remunerações entre funcionários da mesma categoria (por exemplo: auxiliar concursado e auxiliar cooperativado) ( ) sim ( ) não 
9.4 Desenvolvimento de Capacidade

- A atividade exercida é compatível com o seu maior nível de formação?

( ) $\operatorname{sim}($ ) não

- Desenvolve o seu trabalho com autonomia? ( ) sim ( ) não

- Participa das decisões no seu trabalho? ( ) sim ( ) não

- Você realiza várias atividades ao mesmo tempo? ( ) sim ( ) não

- Há retorno da chefia com relação ao seu desempenho? ( ) sim ( ) não

- Você realiza atividade de crescimento profissional (treinamento em serviço, congressos, cursos e outros)? ( ) $\operatorname{sim}($ ) não

9.5 Relacionamento Interpessoal

- Relacionamento interpessoal é favorável ao seu desempenho profissional?

( ) $\operatorname{sim}(\quad)$ não

- Vida familiar é: ( ) satisfatória ( ) insatisfatória

- Possui atividades de lazer? ( ) sim ( ) não

- Quando há dificuldades durante alguma atividade você recebe apoio dos colegas (p. ex. durante a mudança de decúbito) ( ) sim ( ) não

- Existem normas e regulamentos acessíveis (são de fácil acesso? Se houver, é claro) ( ) sim ( ) não

- O trabalho ocupa todo o tempo de sua jornada de trabalho? ( ) sim ( ) não

- O trabalho Ihe proporciona realização? ( ) sim ( ) não 